\title{
A Case of Severe Combined Immunodeficiency Missed by Newborn Screening
}

\author{
Maleewan Kitcharoensakkul ${ }^{1,2}$ (D) - Jahnavi Aluri ${ }^{1} \cdot$ Ibrahim Elsharkawi $^{3} \cdot$ Ashley Steed $^{4} \cdot$ Christopher D. Putnam $^{5,6}$. \\ Anil K. Swayampakula ${ }^{7} \cdot$ Megan A. Cooper $^{1}$. Jeffrey J. Bednarski ${ }^{8}$
}

Received: 3 November 2020 / Accepted: 4 March 2021 / Published online: 12 March 2021

(C) The Author(s), under exclusive licence to Springer Science+Business Media, LLC, part of Springer Nature 2021

To the Editor.

Severe combined immunodeficiency (SCID) is a lifethreatening immune disorder affecting nearly 1:65,000 births in the USA [1]. The SCID newborn screen by T Cell Receptor Excision Circles (TRECs) has an excellent performance with nearly $100 \%$ sensitivity in detecting typical SCID. However, case reports have highlighted missed SCID diagnoses due to false-negative normal newborn screening results [1]. Here we report a young child with a normal SCID newborn screen who presented with two episodes of severe respiratory failure before diagnosis of leaky SCID secondary to a variant in IL2RG, p.Arg222Ser. This variant was previously reported in a SCID patient who presented later in life with recurrent infections and molluscum contagiosum [2]. Functional studies here provide additional insight into the effects of this variant on protein function.

A previously healthy 1 year old African-American male, born full term without complications, initially presented with respiratory failure requiring ECMO at an outside hospital for 9 days. A respiratory viral polymerase chain reaction (PCR) was positive for rhinovirus and adenovirus. He was intubated for 1 month, hospitalized for a total of 6 weeks, and ultimately discharged home. At 15 months, he was rehospitalized for respiratory failure with adenovirus and

Maleewan Kitcharoensakkul

maleewan@wustl.edu

1 Department of Pediatrics, Division of Rheumatology/Immunology, Washington University School of Medicine, Campus box 8116, One Children's Place, Saint Louis, MO 63110, USA

2 Department of Pediatrics, Division of Allergy and Pulmonary Medicine, Washington University School of Medicine, St. Louis, MO, USA

3 Department of Pediatrics, Division of Genetics, Washington University School of Medicine, St. Louis, MO, USA coronavirus OC43 detected by PCR from tracheal aspirates. He had a normal TREC screen at birth, 440 copies/mcl (reference $\geq 250$ ). His immunizations were up-to-date through 6 months of age, including live rotavirus vaccines. There was no family history of immunodeficiency. He had one full 4-year-old sister and a paternal half-brother, both of whom were healthy. Chest radiographs revealed bilateral groundglass opacities (Supplemental Fig. 1a). He was treated with vancomycin, meropenem, and methylprednisolone. One month after hospitalization, he continued to require ventilator support and a chest CT scan revealed patchy consolidations bilaterally (Supplemental Fig. 1b). Upon transfer to our institution, his physical exam revealed poor growth with weight, length, and head circumference at the 7th, 3rd, and 1 st percentile for age, respectively. The lungs exhibited bronchial breath sounds. There was no lymphadenopathy or hepatosplenomegaly. Bronchioalveolar lavage (BAL) was positive for adenovirus and coronavirus OC43. Direct fluorescent antibody staining for Pneumocystis jiroveci was positive. Cultures from BAL and blood for acid-fast bacilli and fungus were negative. His cerebrospinal fluid and blood were both positive for adenovirus with a viral load of 664,000 copies $/ \mathrm{mcl}$ in the blood. Testing for CMV, HSV, and HHV-6 was negative.

4

Department of Pediatrics, Division of Critical Care Medicine, Washington University School of Medicine, St. Louis, MO, USA

5 Department of Medicine, University of California School of Medicine, San Diego, La Jolla, CA, USA

6 San Diego Branch, Ludwig Institute for Cancer Research, La Jolla, CA, USA

7 Department of Pediatrics, Division of Critical Care Medicine, University at Buffalo (SUNY), Buffalo, NY, USA

8 Department of Pediatrics, Division of Hematology/Oncology, Washington University School of Medicine, St. Louis, MO, USA 
Laboratory evaluation on admission revealed normal absolute lymphocyte count of $6110 / \mu 1$, and elevated neutrophil count of $24,150 / \mu l$. Hemoglobin and platelets were normal. Lymphocyte subsets analysis obtained 3 days after admission showed severe $\mathrm{CD}^{+} \mathrm{T}$ and $\mathrm{CD} 16^{+} / \mathrm{CD} 56^{+} \mathrm{NK}$ cell lymphopenia with elevated $\mathrm{CD} 19^{+} \mathrm{B}$ cells. $\mathrm{CD} 4^{+} / \mathrm{CD} 45 \mathrm{RA}^{+}$naïve $\mathrm{T}$ cell numbers were low (Fig. 1a). The percentage of CD3+ HLA-DR+ T cells was normal for age $(4.2 \%)$. HIV PCR was negative. IgG was low with near-normal IgM and normal IgA levels. IgG to tetanus was detectable, but IgG to Haemophilus influenzae was absent. TREC analysis was slightly low at 6610 copies per $10^{6} \mathrm{~T}$ cells ( $\mathrm{ref} \geq 6794$ ). $\mathrm{T}$ cell proliferation showed low viability with impaired $\mathrm{T}$ cell response to phytohemagglutinin, but normal to pokeweed. This study was obtained while the patient was receiving systemic steroids for treatment of PJP. T cell receptor (TCR) spectratyping was abnormal, with 19 of 28 probes demonstrating an oligoclonal distribution and 8 with a polyclonal nonGaussian distribution. Clinical whole genome sequencing (PerkinElmer®) demonstrated a hemizygous missense variant in $I L 2 R G$ c. $664 \mathrm{C}>\mathrm{A}$, resulting in substitution of an arginine at amino acid position 222 with serine (p.Arg222Ser). This variant is absent in gnomAD with allele coverage of at least 183,383 . The CADD PHRED score is 23.6 , indicative of pathogenicity [3]. No other pathogenic variants in disease-causing genes were identified.

The immunological phenotype of low $\mathrm{T}$ and NK cells with normal B cells and an identified variant in IL2RG supported the diagnosis of X-linked SCID (XL-SCID) [4]. Functional testing of the patient's T cells demonstrated absent STAT5 phosphorylation compared to healthy control $\mathrm{T}$ cells following stimulation with IL-2 (Fig. 1b) and IL-15 (not shown), confirming loss of IL-2 signaling which requires IL-2R $\gamma$ and IL2R $\beta$. Interestingly, patient T cells did exhibit STAT5 phosphorylation upon stimulation with IL-7, a cytokine important for T cell differentiation that signals through IL-2R $\gamma /$ IL-7R $\alpha$, although they had a lower response than T cells from healthy control samples (Fig. 1b). The percentage of $\mathrm{CD}^{+}$ recent thymic emigrants as defined by expression of CD31 was low compared to a healthy age-matched control (Supplemental Fig. 2). Written informed consent was obtained for research and approved by the authors' Institutional Review Board.

Trimethoprim-sulfamethoxazole, cidofovir, and immunoglobulin replacement was initiated. Due to nephrotoxicity, cidofovir was switched to brincidofovir. His respiratory status worsened and he was re-cannulated for ECMO. Given his clinical status, he underwent matched-related donor HCT without conditioning at 18 months of age. Two weeks after transplant, he received multivirus-specific $\mathrm{T}$ cells (NCT03475212) for treatment of the adenovirus infection. Due to persistent adenovirus viremia, he was given a second infusion of multivirus-specific T cells 6 weeks after the initial infusion. His post-transplant course was complicated by pneumoperitoneum, acute renal failure from nephrotoxic agents, hydrocephalus requiring ventriculoperitoneal shunt, shunt infection, and failure to engraft donor cells requiring second HCT with reinfusion of matched-related donor cells following preparative regimen with busulfan and fludarabine. Two weeks after his second HCT, his respiratory status improved and he was decannulated from ECMO. Blood adenovirus PCR became undetectable 2 weeks after the second transplant. He underwent tracheostomy and was discharged on a home ventilator after 12 months of hospitalization. Variant testing on his mother confirmed carrier status, and genetic counseling was provided.

Our case highlights an important clinical lesson for immunologists that although newborn screening by TREC assay on dried blood spots is highly sensitive in detecting SCID, falsenegative cases do occur given residual $\mathrm{T}$ cell production in both leaky SCID and SCID with late-onset manifestations [1]. In addition to our case, two other cases of SCID missed by newborn screening have been reported in California [1]. One patient presented with $P J P$ pneumonia and was diagnosed with a $I L 2 R G$ missense variant resulting in p.Arg222Cys. The other patient had ADA deficiency and was diagnosed with SCID at 23 months of age after having recurrent infections.

The Arg222Ser variant of $I L 2 R G$ gene was previously described in one patient, although that infant was born prior to newborn screening and there was no analysis of TREC at birth [2]. That case had later-onset of recurrent infections at 5 years of age without history of Pneumocystis infection despite profound $\mathrm{CD}^{+} \mathrm{T}$ cell lymphopenia, and he had normal NK cell count and IgG level. There were at least 20 cases of Arg222Cys giving rise to clinical phenotypes ranging from leaky SCID with later onset of manifestations to typical SCID [5]. The majority of Arg222Cys patients had variable $\mathrm{CD} 3+\mathrm{T}$ cell count and proliferative responses to mitogen. Of 3 patients whom TRECs obtained during neonatal period, 2 were normal and 1 had slightly reduced TREC levels [1, 6, 7].

The significantly reduced naïve $\mathrm{CD} 4+\mathrm{T}$ cells despite slightly low TREC in our patient could be due to impaired IL-2 mediated peripheral $\mathrm{T}$ cell hemostasis as demonstrated in previous studies of $\operatorname{Arg} 222 \mathrm{Cys}$ variant [6]. Interestingly, our patient's $\mathrm{T}$ cells had a measurable response to IL-7 stimulation, suggesting partially intact IL-2R $\gamma / \mathrm{IL}-7 \mathrm{R} \alpha$ signaling and potentially explaining the presence of naïve $\mathrm{T}$ cells. The mechanism underlying the differences between partiallyintact IL-7 but deficient IL-2/-15 (IL-2R $\gamma / \mathrm{IL}-2 \mathrm{R} \beta$ ) signaling is uncertain. Amino acid Arg222 is within the IL-2R $\gamma$ tryptophan/arginine ladder [8] and not at the interface with IL-2R $\beta$, but the alteration of this structure may control the hinge loop that forms part of the interleukin binding surface (Supplemental Fig. 3). The Arg222 side chain is sandwiched between Trp179 and Trp240 in the trypophan/arginine ladder, 
Fig. 1 Immune phenotype and STAT5 phosphorylation in patient's $T$ cells. a Immune assessments of our patient at 15 months of age on presentation at second hospitalization.

Reference ranges for age shown. (*age-reference values of T cell subsets for 1-2 years old, Shearer et al. JACI. 2003;112:973-80.

*Age-reference values of B cell subsets for 6-18 months old, Duchamp M, et al. Immunity, Inflammation and

Disease.2014;2:131-140). Abbreviations: TREC, T cell receptor excision circle; PHA, phytohemagglutinin. b Phosphorylation of STAT5 in $\mathrm{CD} 3+\mathrm{T}$ cells after incubation with IL-7 (100 ng/mL) for $30 \mathrm{~min}$, or IL-2 $(100 \mathrm{U} / \mathrm{mL})$ for $15 \mathrm{~min}$, on patient and healthy control. Stimulated and unstimulated conditions are shown in blue and red, respectively. c Architecture of the human IL-2 signaling complex and the position of $\operatorname{Arg} 222$ on IL2RG a

\begin{tabular}{|l|c|c|}
\hline \multicolumn{1}{|c|}{ Laboratory } & Results & Ref range \\
\hline Lymphocyte subpopulation & & \\
CD3+ T cells (cells/ $\mu$ l) & 384 & $2100-6200$ \\
CD4+ T cells (cells/ $\mu$ l) & 265 & $1300-3400$ \\
CD8+ T cells (cells/ $\mu$ l) & 114 & $620-2000$ \\
CD19+ B cells (cells/ $\mu \mathrm{l}$ ) & 50 & $720-2600$ \\
CD16+ CD56+ NK cells (cells/ $\mu$ l) & 6610 & $180-920$ \\
TREC (copies per 106 CD3 T cells) & 180 & $1100-3700$ \\
CD4+CD45RA+ naïve T cells (cells/ $\mu$ l) & 70 & $64-93$ \\
\%CD45RA+ of CD4+ T cells & 0.8 & $3.5-12.2$ \\
\%CD27+ of CD19+ B cells & 0.4 & $0.6-3.7$ \\
\%CD27+ IgM-IgD- of CD19+ B cells & 37.8 & $\geq 75$ \\
\hline Lymphocyte proliferation to mitogen & 1.8 & $\geq 4.5$ \\
Viability of lymphocytes at day 0 (\%) & 18.6 & $\geq 3.5$ \\
Max proliferation of Pokeweed as \% CD45+ cells & 0.6 & $\geq 3.9$ \\
Max proliferation of Pokeweed as \% CD3+ cells & 7.5 & $\geq 49.9$ \\
Max proliferation of Pokeweed as \%CD19+ cells & 36.7 & $\geq 58.5$ \\
Max proliferation of PHA as\%CD45+ cells & & \\
Max proliferation of PHA as \%CD3+ cells & 113 & $475-1210$ \\
\hline Immunoglobulins & 38 & $41-183$ \\
IgG (mg/dl) & 84 & $21-291$ \\
IgM (mg/dl) & 0.06 & $\geq 0.01$ \\
IgA (mg/dl) & Undetectable & $\geq 0.15$ \\
\hline Vaccine titers & & \\
Tetanus IgG (IU/mL) &
\end{tabular}

b

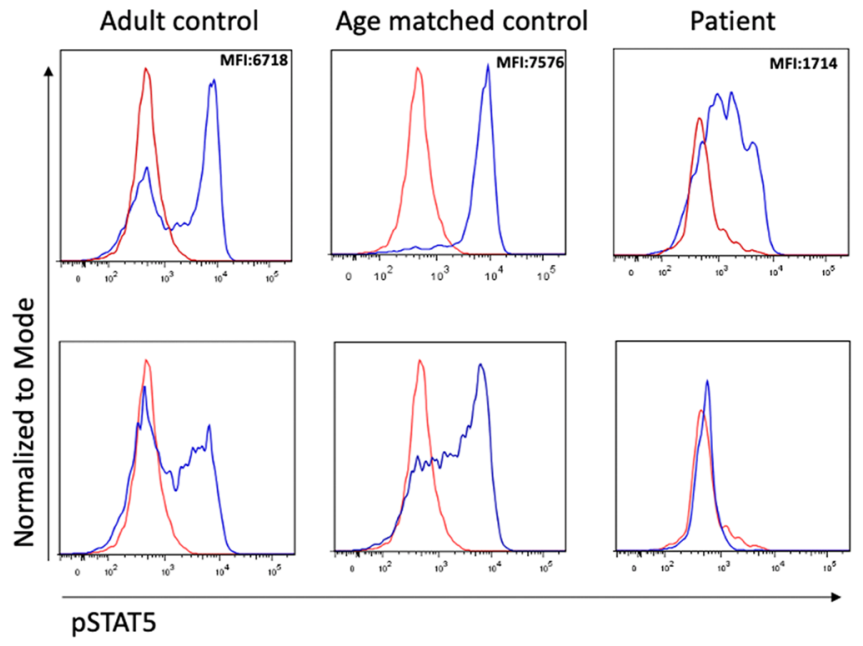

IL-7 (100ng/mL) stimulation

IL-2 (100U/mL) stimulation

C

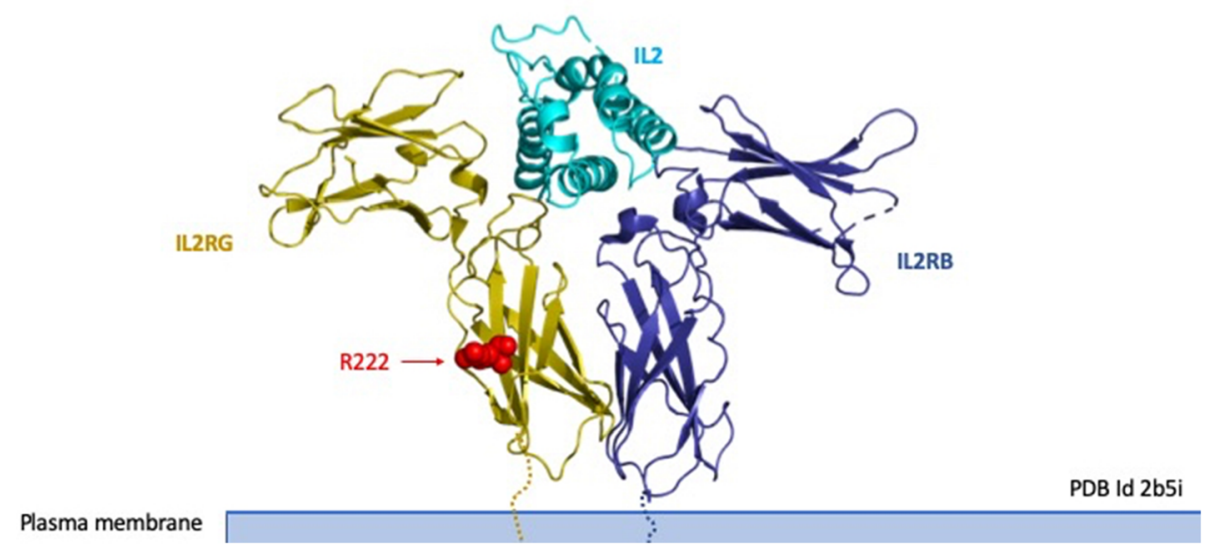


suggesting the more polar p.Arg222Ser mutation disrupts the structure more than the p.Arg222Cys mutation. There is currently no structure of IL-2R $\gamma / \mathrm{IL}-7 \mathrm{R} \alpha$; however, studies in our patient here suggest that p.Arg222Ser alters IL-7 binding and/ or association with IL-7R $\alpha$ to a lesser extent than IL-2. Additional studies on impact of an alteration of Arg222 and other protein changes in IL-2R $\gamma$ identified in patients, particularly with "leaky" SCID, will improve our understanding of this important receptor and its partners.

Overall, our case and these previously reported patients highlight that hypomorphic IL2RG variants can lead to leaky $\mathrm{T}$ cell development/function that can result in normal TRECs at birth, and even in our case at diagnosis, and potential missed SCID diagnoses on newborn screening with disease presentation later in life.

The sequalae of delayed SCID diagnosis is paramount as outcomes of SCID are intimately tied to timing and infection status at the time of HCT. A delay in diagnosis can result in permanent organ damage or death in SCID patients. Our patient remarkably recovered from two prolonged episodes of respiratory failure but continues to require invasive ventilatory support and complex care.

Our case highlights the importance for immunologists to keep a high index of suspicion for SCID in children with severe/opportunistic infections even in those with normal newborn SCID screen.

Supplementary Information The online version contains supplementary material available at https://doi.org/10.1007/s10875-021-01020-8.

Acknowledgements Research support was provided by St. Louis Children's Hospital, Center for Pediatric Immunology, and The Jeffrey Modell Diagnostic and Research Center for Primary Immunodeficiencies at St. Louis Children's Hospital. We thank Michael Pulsipher M.D. (Cancer and Blood Disease Institute, Kerk School of Medicine of University of Southern California), Catherine M Bollard, M.D. (Center for Cell and Gene Therapy, Baylor College of Medicine), and Michael D Keller, M.D. (Division of Allergy \& Immunology, Children's National Health System) for assistance with multivirus-specific T-cells for treatment of systemic adenovirus infection in our patient. We also thank Dr. Amir Sadhigi (Mayo clinic laboratories) for assistance with reviewing and repeating TREC assays.
Funding The Jeffrey Modell Diagnostic and Research Center for Primary Immunodeficiencies at St. Louis Children's Hospital and the Center for Pediatric Immunology at Washington University, Department of Pediatrics.

\section{Declarations}

Competing Interests The authors declare no competing interests.

\section{References}

1. Amatuni GS, Currier RJ, Church JA, Bishop T, Grimbacher E, Nguyen AA, et al. Newborn screening for severe combined immunodeficiency and T-cell lymphopenia in California, 2010-2017. Pediatrics. 2019;143(2):e20182300.

2. Thrasher AJ, Hacein-Bey-Abina S, Gaspar HB, Blanche S, Davies EG, Parsley K, et al. Failure of SCID-X1 gene therapy in older patients. Blood. 2005;105(11):4255-7.

3. Kircher M, Witten DM, Jain P, O'Roak BJ, Cooper GM, Shendure J. A general framework for estimating the relative pathogenicity of human genetic variants. Nat Genet. 2014;46(3):310-5.

4. Lek M, Karczewski KJ, Minikel EV, Samocha KE, Banks E, Fennell T, et al. Analysis of protein-coding genetic variation in 60,706 humans. Nature. 2016;536(7616):285-91.

5. Lim CK, Abolhassani H, Appelberg SK, Sundin M, Hammarstrom L. IL2RG hypomorphic mutation: identification of a novel pathogenic mutation in exon 8 and a review of the literature. Allergy Asthma Clin Immunol. 2019;15:2.

6. Fuchs S, Rensing-Ehl A, Erlacher M, Vraetz T, Hartjes L, Janda A, et al. Patients with $\mathrm{T}(+) /$ low $\mathrm{NK}(+) \mathrm{IL}-2$ receptor gamma chain deficiency have differentially-impaired cytokine signaling resulting in severe combined immunodeficiency. Eur J Immunol. 2014;44(10): 3129-40.

7. Stepensky P, Keller B, Shamriz O, von Spee-Mayer C, Friedmann D, Shadur B, et al. $\mathrm{T}(+) \mathrm{NK}(+)$ IL-2 receptor gamma chain mutation: a challenging diagnosis of atypical severe combined immunodeficiency. J Clin Immunol. 2018;38(4):527-36.

8. Stauber DJ, Debler EW, Horton PA, Smith KA, Wilson IA. Crystal structure of the IL-2 signaling complex: paradigm for a heterotrimeric cytokine receptor. Proc Natl Acad Sci U S A. 2006;103(8):2788-93.

Publisher's Note Springer Nature remains neutral with regard to jurisdictional claims in published maps and institutional affiliations. 